\title{
Comunicação entre o profissional da nutrição e o paciente com surdez
}

\section{Communication between the nutritionist and the deaf patient}

Larissa Rayane Sousa Sousa ${ }^{1}$, Naylanne Lima de Sousa ${ }^{2}$, Luciana Pereira Pinto Dias $^{3}$, Lívia Muritiba Pereira de Lima Coimbra ${ }^{4}$

\section{RESUMO}

Inclusão social no atendimento aos portadores de necessidades especiais é um fator necessário para um serviço de qualidade e entender o relacionamento entre o paciente surdo e o profissional de saúde torna-se essencial. O presente trabalho objetivou-se avaliar a qualidade da comunicação entre o profissional nutricionista e paciente com surdez, através de estudo transversal descritivo quali-quantitativa, realizado em três hospitais da cidade de São Luís-MA, com 40 pessoas portadoras de surdez e 20 nutricionistas. Foram utilizados questionários semiestruturados e os dados coletados tabulados no Programa Microsoft Excel. Foi visto que, 55,6\% dos nutricionistas já tiveram contato com paciente surdo no local de trabalho, a maioria $(22,2 \%)$ declarou atender através de um intérprete, a minoria $(5,6 \%)$ através da língua portuguesa e nenhum através da LIBRAS. Os nutricionistas $(83,3 \%)$ admitiram não estarem aptos a atender pessoas com surdez, já as pessoas com surdez, todos já receberam atendimento nutricional e a maioria $(33,3 \%)$ através da língua portuguesa escrita, gestos e mímicas, a minoria (13,3\%) através da língua portuguesa escrita e nenhum que soubesse LIBRAS. Com isso, foi possível observar a dificuldade dos nutricionistas na comunicação com as pessoas com surdez, devido a falha comunicativa, devido ao não conhecimento em LIBRAS.

Palavras-chave: Surdez. Nutrição. Comunicação. Atendimento Humanizado.

\section{ABSTRACT}

Social inclusion in the care of people with special needs is a necessary factor for a quality service and understanding the relationship between the deaf patient and the health professional is essential. The present study aimed to evaluate the quality of communication between the professional nutritionist and the patient with deafness, through a descriptive cross-sectional quali-quantitative study, carried out in three hospitals in the city of São Luís - MA, with 40 people with deafness and 20 nutritionists. Semi-structured questionnaires were used and the data collected were tabulated in the Microsoft Excel program. It was seen that $55.6 \%$ of nutritionists have already had contact with a deaf patient in the workplace, the majority $(22.2 \%)$ declared to attend through an interpreter, the minority (5.6\%) through the Portuguese language and none through LIBRAS. Nutritionists (83.3\%) admitted that they were not able to serve people with deafness, while people with deafness, all have already received nutritional care and the majority (33.3\%) through the written Portuguese language, gestures and mimics, the minority (13.3\%) through the written Portuguese language and none who knew LIBRAS. With that, it was possible to observe the difficulty of nutritionists in communicating with people with deafness, due to communicative failure, due to the lack of knowledge in LIBRAS.

Keywords: Deafness. Nutrition. Communication. Humanized Service.

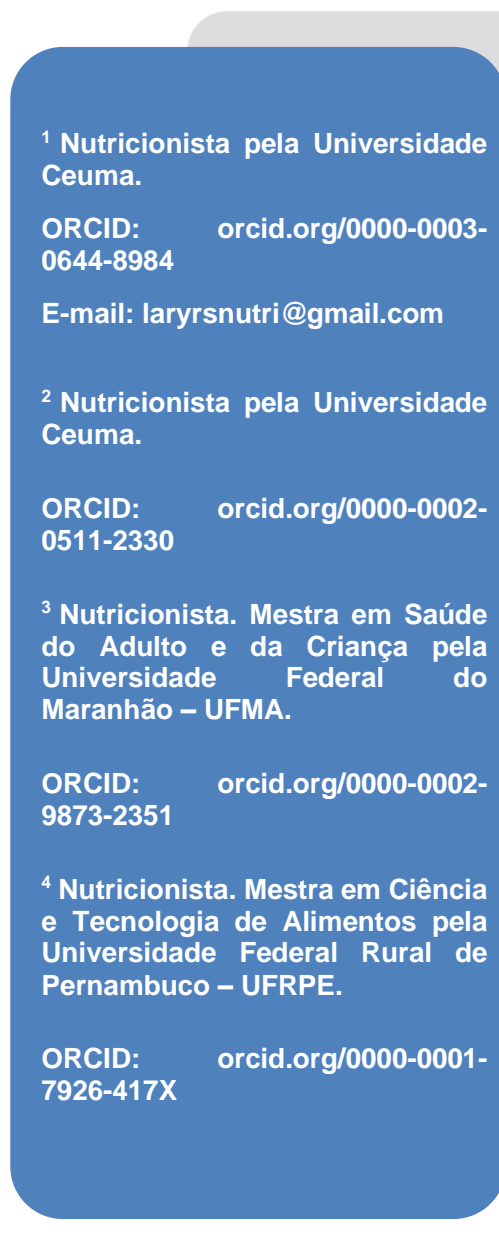

${ }^{1}$ Nutricionista pela Universidade Ceuma.

0644-8984

E-mail: laryrsnutri@gmail.com

${ }^{2}$ Nutricionista pela Universidade Ceuma.

ORCID:

0511-2330

${ }^{3}$ Nutricionista. Mestra em Saúde do Adulto e da Criança pela Universidade do

ORCID:

9873-2351

${ }^{4}$ Nutricionista. Mestra em Ciência e Tecnologia de Alimentos pela Universidade Federal Rural de Pernambuco - UFRPE.

ORCID:

7926-417X 


\section{INTRODUÇÃO}

Segundo a Organização Mundial de Saúde (OMS), no mundo, existem cerca de 600 milhões de pessoas com deficiência. Dados do Censo Demográfico de 2010 estimam que haja 45,6 milhões de pessoas com deficiência no Brasil, sendo que 9,7 milhões de brasileiros apresentam deficiência auditiva, destas, 7,6\% correspondem às pessoas que têm perda auditiva severa (BRASIL, 2012).

O Decreto ํㅜ 5.626, de 22 de dezembro de 2005, Art. 2º, menciona a diferença entre deficiência auditiva que corresponde à perda bilateral, parcial ou total, de quarenta decibéis ou mais, e a surdez, que devido à perda auditiva, a pessoa interage e compreende o mundo através de experiências visuais, apresentando sua cultura principalmente pelo uso da Língua Brasileira de Sinais - LIBRAS (BRASIL, 2005).

A comunicação é fundamental para o convívio, através dela, podem-se compartilhar ideias, emoções e sentimentos, por isso, é necessária uma boa comunicação para compreender, perceber e traduzir, de forma cuidadosa e responsável o que aflige o paciente (CAVAGNA et al., 2019).

O encontro dos profissionais da saúde com o paciente surdo acontece fora dos padrões previstos na rotina de qualquer profissional, a situação torna-se ainda mais limitante quando o profissional não tem conhecimento sobre a linguagem de LIBRAS, dificultando a criação de um vínculo com o paciente (SOUZA et al., 2017).

A falta de comunicação dificulta um atendimento humanizado, já que a inclusão social no atendimento aos portadores de necessidade especiais, é um fator necessário para um serviço de qualidade, logo, entender o relacionamento entre o paciente surdo e 0 profissional é uma conjunção essencial para qualificar os serviços prestados a essa comunidade (SOUZA; PORROZZI, 2017).

Tendo o conhecimento sobre alimentação e nutrição a população consegue promover hábitos alimentares mais saudáveis. Porém, pacientes surdos poderão não ter informações sobre alimentação da forma coerente caso os profissionais habilitados a tal função não conseguirem se comunicar do modo correto com estes pacientes (SANTOS; BELGOLD, 2018).

Quando se tem um bloqueio de comunicação entre o profissional e o paciente, a promoção da saúde fica comprometida. Como o paciente surdo não expressa seus problemas de forma compreensível, o profissional fica impossibilitando de mostrar seus conhecimentos, fazendo com que o atendimento não seja eficaz (SILVA; ANDRADE, 2018). 
Tudo isso poderia ser resolvido com um interprete, porém, o atendimento seria através de um intermediário, e assim não seria estabelecido um vínculo direto com o paciente, além de existir vários desafios, como a dificuldade de encontrar um interprete disponível, a desconfiança do surdo em relação ao interprete, e o paciente não se sentir à vontade para se expor (GOMES et al., 2017; CUNHA; PEREIRA; OLIVEIRA, 2019).

Portanto, a presente pesquisa teve como objetivo avaliar se a comunicação entre o profissional nutricionista e o paciente com surdez está sendo realizada de forma adequada, viabilizando um bom atendimento.

\section{MATERIAIS E METODOS}

Trata-se de um estudo transversal descritivo, com abordagem quali-quantitativa, realizado no mês de setembro de 2018. Seguindo as recomendações da Resolução no 466 de 12 de dezembro de 2012 do Conselho Nacional de Saúde, incialmente, a pesquisa foi submetida e aprovada sob o número de parecer 2.851 .588 pelo Comitê de Ética em Pesquisa da Universidade CEUMA.

A pesquisa foi desenvolvida em três hospitais da cidade de São Luís - MA, sendo um dos hospitais, referência em urgência e emergência para todo o Estado do Maranhão, o outro atende tanto ao Sistema Único de Saúde (SUS) quanto aos planos de saúde, por ser o único hospital no Maranhão que contém equipamentos e serviços especializados no tratamento do câncer, já o terceiro é um dos hospitais particulares mais utilizados pela população de São Luís - MA. Além dos hospitais a coleta de dados também aconteceu em um Centro que atende pessoas com surdez.

Participaram do presente estudo, 40 pessoas com surdez e 20 nutricionistas selecionados aleatoriamente. Foram incluídos na pesquisa profissionais da nutrição com idade igual ou superior a 20 anos, com mais de um ano atuando como nutricionista da área clínica, de ambos os sexos e pessoas com surdez com idade igual ou superior a 16 anos, de ambos os sexos, que são atendidas no referido centro de apoio as pessoas com surdez.

Para a coleta de dados aplicou-se questionários semiestruturados aos nutricionistas e às pessoas com surdez. O roteiro percorreu os seguintes tópicos: I - Dados sócio demográficos (idade, escolaridade, tempo de trabalho); II - Relato do conhecimento do nutricionista sobre a abordagem ao paciente com surdez; III - Relato do atendimento e das dificuldades encontras pelos surdos no atendimento nutricional e IV - Descrição do processo de comunicação entre as partes envolvidas. O questionário foi auto administrado para nutricionistas e para os surdos, as perguntas foram realizadas por um interprete. 
Os dados coletados foram tabulados com auxílio do Programa Microsoft Excel 2010, para análise estatística descritiva e interpretação dos resultados, as variáveis foram categorizadas, descritas por meio de suas frequências absolutas (n) e relativas (\%), sendo posteriormente apresentadas em forma de tabelas.

\section{RESULTADOS E DISCUSSÃO}

Em relação aos nutricionistas entrevistados, $72,2 \%$ tinham mais de 30 anos, 50\% atuavam na área clínica entre 5 a 10 anos, metade (50\%) eram graduados em universidades particulares e a grande maioria $(88,9 \%)$ apresentava pós-graduação em nutrição (Tabela 1).

Tabela 1 - Perfil dos nutricionistas entrevistados.

\begin{tabular}{lc}
\hline Variável & $\%$ \\
\hline Faixa etária & \\
Entre 20 e 30 anos & $27,8 \%$ \\
Mais de 30 anos & $72,2 \%$ \\
Formação & \\
Graduado em nutrição & $11,1 \%$ \\
Pós-Graduado & $88,9 \%$ \\
Instituição formadora & \\
Pública Estadual & $5,6 \%$ \\
Publica Federal & $44,4 \%$ \\
Particular & $50 \%$ \\
Tempo de atuação na área clínica & \\
de 1 a 5 anos & $22,2 \%$ \\
de 5 a 10 anos & $50 \%$ \\
mais de 10 anos & $27,8 \%$ \\
\hline
\end{tabular}

Fonte: Construção do Autor

A grande maioria $(77,8 \%)$ dos nutricionistas relataram ter informação sobre pessoas com deficiência, contudo, esse percentual reduziu para 38,9\% quando a informação foi em relação ao indivíduo com surdez, apesar dessa porcentagem ser baixa, mais da metade $(55,6 \%)$ dos nutricionistas entrevistados já tiveram contato com o paciente surdo no local de trabalho (Tabela 2).

Mesmo o percentual dos nutricionistas entrevistados que já tiveram contato com o paciente surdo no local de trabalho ser de $55,6 \%, 22,2 \%$ dos nutricionistas declararam 
atender através de um intérprete, 16,7\% através de gestos e mímica, 11,1\% através da língua portuguesa escrita e 5,6\% através da língua portuguesa e nenhum através da linguagem de LIBRAS (Tabela 2).

Em relação aos que ainda não tiveram contato com pacientes surdos o modo relatado como provavelmente atenderia foi através da língua portuguesa escrita $(16,7 \%)$ e a minoria afirmou que atenderia através de um intérprete (5\%), nenhum relatou que usaria a LIBRAS (Tabela 2).

Em uma pesquisa realizada com 32 participantes (agente administrativo, assistente social, coordenador técnico, dentista, enfermeiro, fisioterapeuta, fonoaudiólogo, porteiros, nutricionista, profissional de educação física, pedagogo, psicólogo, técnico em enfermagem e terapeuta ocupacional) atuantes em unidade pública regional de reabilitação física, situada no Rio de Janeiro, apenas 12,5\% tinham domínio sobre a LIBRAS e faziam uso para se comunicar com os pacientes surdos, os outros relataram recorrer aos colegas de trabalho que conhecem a LIBRAS, fazer uso da escrita, gestos, mímicas e leitura labial (MACHADO et al., 2015).

Há uma grande diferença entre a LIBRAS (visual-espacial) e a língua portuguesa (oralauditiva), a língua portuguesa contém escrita alfabética e sintaxe linear, em contrapartida a LIBRAS apresenta estrutura tópico-comentário, por essa razão a forma escrita pode não ser um meio de comunicação eficaz (SOUZA et al., 2017).

Esse quadro poderia ser diferenciado se as instituições acadêmicas proporcionassem aos futuros profissionais o ensino da LIBRAS, dando noções básicas sobre a língua de sinais e posicionamento frente a essa população, trazendo um novo olhar e despertando um maior interesse por parte dos estudantes em relação a esses indivíduos (GOMES et al., 2017).

Vale ressaltar que além de aprender a LIBRAS, os profissionais necessitam estar sempre atualizados quanto a troca de informações com os surdos, aprofundando seus conhecimentos constantemente com cursos mais avançados e específicos de LIBRAS na saúde (CUNHA; PEREIRA; OLIVEIRA, 2019). 
Tabela 2 - Conhecimento dos nutricionistas sobre a abordagem ao paciente com surdez.

\begin{tabular}{lc}
\hline Variável & $\%$ \\
\hline Informação sobre a pessoa com deficiência & \\
Sim & $77,8 \%$ \\
Não & $22,2 \%$ \\
Informação sobre a pessoa com surdez & \\
Sim & $38,9 \%$ \\
Não & $61,1 \%$ \\
Contato com pessoas surdas dentro do local de trabalho & \\
Sim & $55,6 \%$ \\
Não & $44,4 \%$ \\
Atendimento ao paciente surdo & \\
Através da língua portuguesa & $5,6 \%$ \\
Attavés da língua portuguesa escrita & $11,1 \%$ \\
Através de gestos e mímica & $16,7 \%$ \\
Através de umm interprete & $22,2 \%$ \\
Através da língua brasileira de sinais - LIBRAS & $0,0 \%$ \\
Atenderia o paciente surdo & \\
Através da língua portuguesa & $11,1 \%$ \\
Através da língua portuguesa escrita & $22,2 \%$ \\
Através de gestos e mímica & $11,1 \%$ \\
Através de um interprete & $16,7 \%$ \\
Através da língua brasileira de sinais - LIBRAS & $0,0 \%$ \\
Nutricionistas aptos a atender pessoa com surdez & \\
Sim & $16,7 \%$ \\
Não & $83,3 \%$ \\
Conhecimento sobre LIBRAS & \\
Sim & $22,2 \%$ \\
Não & $77,8 \%$ \\
Acredita na sociedade inclusiva & \\
Sim & $100 \%$ \\
Não & $0,0 \%$ \\
\hline & \\
\hline &
\end{tabular}

Fonte: Construção do Autor

Todos os nutricionistas entrevistados acreditam em uma sociedade inclusiva. Contudo, é preciso trazer essa minoria para o contexto social, uma forma disso acontecer é entender que a língua de sinais tem a mesma relevância que a língua oral, e ela precisa estar nos ambientes de circulação pública e privada (ARAÚJO; MENEZES; ARAÚJO, 2017).

Foi relatado por parte dos nutricionistas que a prefeitura da cidade de São Luís oferece curso de LIBRAS para os profissionais da área da saúde, porém, os mesmos não apresentaram interesse em realizá-lo.

Um dos nutricionistas referiu-se sobre a comunidade surda da seguinte maneira: "o atendimento a essas pessoas acontece com menos frequência, não vejo necessidade de 
me qualificar tanto, quase sempre vêm acompanhados, e os acompanhantes dizem tudo que há com o paciente".

O diálogo é uma ferramenta determinante em todo o processo de promoção e reabilitação à saúde, quando tal não é efetuada de modo adequado, há um comprometimento pela ausência de entendimento entre as partes envolvidas (JALLES; SANTOS; REINALDO, 2017).

Segundo França et al. (2016), os profissionais da saúde aptos a se comunicar de forma correta, estão integrando as pessoas com surdez no meio social. Quando essa parcela da população precisa se adaptar para se integrar a sociedade, principalmente em busca da saúde, percebe-se que a população não está preparada para receber o surdo, sua inclusão na sociedade atualmente torna-se um assunto bem discutido, visando a aceitação de um grupo antes marginalizado (GOMES et al., 2017).

Do total de nutricionistas entrevistados apenas $22,2 \%$ referiram conhecer a LIBRAS, alguns relataram conhecer $\mathrm{o}$ alfabeto, outros alguns gestos, mas nenhum dos nutricionistas tem domínio sobre a LIBRAS e $83,3 \%$ admitem que hoje os nutricionistas não estão aptos a atender pessoas com surdez (Tabela 2).

Segundo Gomes et al. (2017), presar pela qualidade de vida dos indivíduos é dever de todo profissional da saúde. Para abranger toda a população, os profissionais devem estar habilitados para atender uma minoria, que são as pessoas com deficiência, igualmente como prestam atendimento às pessoas cujo cuidado não precisa ser diferenciado.

Um dos profissionais da área da saúde diretamente ligado a essa conduta é o nutricionista, que deve atuar defendendo o Direito à Saúde, à Segurança Alimentar e Nutricional e o Direito Humano à Alimentação Adequada, dos indivíduos e da coletividade, tendo respeito à vida, não discriminando qualquer natureza em sua relação profissional (CFN, 2018).

A Tabela 3 demonstra o perfil das pessoas com surdez. Apesar de $50 \%$ dos surdos entrevistados apresentarem faixa etária menor que 20 anos, isso não implicou nos resultados da pesquisa, visto que todos possuem desenvolvimento físico e mental suficiente para interpretar e opinar em relação ao uso da sua primeira língua, a LIBRAS. 
Tabela 3 - Perfil dos surdos e conhecimento sobre a profissão do nutricionista.

\begin{tabular}{lc}
\hline Variável & $\%$ \\
\hline Faixa etária & \\
Menos de 20 anos & $50,0 \%$ \\
Entre 20 e 25 anos & $37,5 \%$ \\
Mais de 30 anos & $12,5 \%$ \\
Escolaridade & \\
Ensino Fundamental Incompleto & $0,0 \%$ \\
Ensino Fundamental Completo & $17,5 \%$ \\
Ensino Médio Incompleto & $50,0 \%$ \\
Ensino Médio Completo & $25,0 \%$ \\
Ensino Superior Incompleto & $2,5 \%$ \\
Ensino Superior Completo & $5,0 \%$ \\
Conhece a profissão do nutricionista & \\
Sim & $37,5 \%$ \\
Não & $62,5 \%$ \\
\hline
\end{tabular}

Fonte: Construção do Autor

O grau de escolaridade dos surdos foi maior ao ensino médio incompleto (50\%) e apenas 5\% apresentava curso superior (Tabela 3 ). Esse baixo percentual em relação ao nível superior pode se dar devido ao preconceito, que é considerado a principal barreira no processo de escolarização dos surdos, na medida que a sociedade ouvinte se mantém afastada e pouco se esforça para estabelecer interação social e comunicação com os mesmos (MOURA; LEITE; MARTINS, 2016).

Em relação ao conhecimento da profissão do nutricionista, 62,5\% informaram não conhecer. Foi possível observar que os mesmos não têm compreensão sobre uma alimentação de qualidade o que pode refletir em futuras doenças causadas pela má alimentação (Tabela 3).

Apenas $37,5 \%$ dos surdos entrevistados conhecem a profissão do nutricionista e, desses todos já receberam atendimento nutricional, sendo 33,3\% através da língua portuguesa escrita, gestos e mímicas, 26,7\% através de gestos e mímicas, 13,3\% através da língua portuguesa escrita e 26,7\% através de um intérprete (Tabela 4).

Para aqueles que já receberam atendimento nutricional a principal dificuldade encontrada quando precisou-se comunicar com o nutricionista foi pela falta de comunicação desses profissionais em LIBRAS (60\%) (Tabela 4).

No estudo realizado por Araújo et al. (2019), ao avaliaram as dificuldades enfrentadas pelos pacientes surdos no atendimento médico em Belo Horizonte - MG, pode ser observado que 68,8 \% dos pacientes não compreendia "nada/quase nada" do seu diagnóstico e/ou procedimentos realizados, e 37,5 \% não compreendia claramente as recomendações e tratamento descrito pelo profissional. 
Tabela 4 - Atendimento e dificuldades encontradas pelos surdos que já tiveram atendimento nutricional.

\begin{tabular}{lc}
\hline Variável & $\%$ \\
\hline Atendimento realizado pelo nutricionista & \\
Através da língua portuguesa & $0,0 \%$ \\
Através da língua portuguesa escrita, gestos e mímicas & $33,3 \%$ \\
Através de gestos e mímicas & $26,7 \%$ \\
Através da língua portuguesa escrita & $13,3 \%$ \\
Através de um interprete & $26,7 \%$ \\
Através da língua brasileira de sinais - LIBRAS & $0,0 \%$ \\
Dificuldades encontradas & \\
A falta de comunicação desse profissional em LIBRAS & $60,0 \%$ \\
Não tem ninguém que sabe LIBRAS no local & $40,0 \%$ \\
Encontrou algum nutricionista que saiba LIBRAS & \\
Sim & $0,0 \%$ \\
Não & $100,0 \%$ \\
Relacionamento com o nutricionista & \\
Regular & $100,0 \%$ \\
\hline
\end{tabular}

Fonte: Construção do Autor

O modo correto de comunicação com esse público (através da LIBRAS), dificilmente é realizado pelos profissionais da área da saúde, como foi possível observar nesta pesquisa, a forma certa de comunicação entre as partes envolvidas não foi estabelecida. Ter um intermediário no processo de comunicação pode distanciar o vínculo com o paciente, visto que as informações em relação a condição de saúde não são repassadas diretamente para o surdo (SILVA; ANDRADE, 2018; CAVAGNA et al., 2019).

Marquete, Costa e Teston (2018), relatam a falta de privacidade, desconfiança e dificuldade em encontrar um intérprete disponível, com isso membros da família poderiam exercer esse papel, porém o paciente surdo pode não se sentir à vontade para se expor, e os familiares, mesmo sem intenção, poderiam alterar o assunto da interlocução, devido à falta de familiarização com os termos da área da saúde utilizados, ou por não serem fluentes em LIBRAS.

Para Araújo et al. (2019), a presença de uma terceira pessoa durante os atendimentos médicos, por um lado pode facilitar a comunicação, no entanto por outro pode levar à falta de privacidade e de autonomia por parte dos pacientes, sendo que muitas vezes o paciente pode omitir informações importantes sobre sua saúde, seja por vergonha ou constrangimento. Os autores afirmam que durante uma consulta é importante o profissional 
de saúde manter um diálogo diretamente com o paciente para se estabelecer vinculo de segurança e afinidade entre ambos.

No estudo de Pires e Almeida (2016), quando avaliado a percepção do surdo sobre o atendimento nos serviços de saúde em Vitória da Conquista - BA, foi relatada a indignação dos surdos com os profissionais da saúde por direcionarem perguntas aos acompanhantes, refletindo um sentimento de insegurança, além de perceberem a falta de interesse dos profissionais com os mesmos no atendimento.

Os pacientes com surdez já atendidos pelos profissionais da nutrição ainda não encontraram um nutricionista que soubesse a LIBRAS (100,0\%), constatando que seu relacionamento com o profissional aconteceu de forma regular (Tabela 4).

O bloqueio de comunicação entre os profissionais da saúde e o indivíduo com surdez precisa ser quebrado para que sejam assistidos de forma global, respeitando suas diferenças. A sociedade necessita mudar os pensamentos em relação a raça, nacionalidade, condições econômicas e preconceito com portadores de deficiência, só assim a promoção à acessibilidade e a inclusão social será estabelecida (KASTRUP; THOMAS, 2018).

Incluir a pessoa com surdez no contexto social vem sendo um assunto bastante abordado nos dias atuais, pois é de extrema importância que tenham os mesmos direitos que os ouvintes, para que possam ter acesso aos serviços de saúde com a mesma qualidade (GUEDES; SILVA, 2017).

Os profissionais serão mais humanistas, críticos e farão atuação inclusiva, quando ampliarem a visão sobre esses indivíduos, entenderem a importância deles na sociedade e se adequarem para obtenção de uma comunicação correta para toda população (MARQUETE; COSTA; TESTON, 2018; ARAÚJO et al., 2019).

Uma das limitações da presente pesquisa foi em relação à amostra, podendo não ser completamente representativa da totalidade de nutricionistas e surdos de São Luís Maranhão. Em contrapartida, são poucos os estudos que abordam esta problemática. Com isto é sugerível que haja a realização de estudos com um maior número de profissionais nutricionistas, também utilizando outras metodologias para analisar as necessidades do Estado do Maranhão e outras regiões do Brasil, relacionando os fatores para enfrentamento desta problemática. 


\section{CONSIDERAÇÕES FINAIS}

É de extrema importância de investimentos em pesquisas com o tema em questão, com o intuito de avaliar a qualidade da comunicação entre o profissional nutricionista e o paciente com surdez durante 0 atendimento nutricional e dessa forma contribuir com conhecimento, trazendo novos desafios e melhores condições para o atendimento desse público, contribuindo para o desenvolvimento de uma sociedade mais inclusiva e justa.

É notável a dificuldade dos nutricionistas em relação à comunicação com as pessoas com surdez, devido à falha na maneira comunicativa, por não disporem do conhecimento em LIBRAS e também foi observada a insatisfação dos surdos com a situação em questão.

O nutricionista possui o intuito de promoção e recuperação da saúde e é o único profissional habilitado para fornecer informações sobre a alimentação, quando não há o modo correto de comunicação, o atendimento fica comprometido. Vale ressaltar que a presença do intérprete simplificaria o processo de comunicação, todavia, de maneira integral não colaboraria com a inclusão social do surdo.

Observando os obstáculos apresentados pelos nutricionistas para a comunicação com o indivíduo surdo, gera a necessidade de ressaltar a importância de investir na qualificação profissional e conscientização para atendimento desse público. Assim, agregar a LIBRAS como disciplina curricular é essencial para se ter uma sociedade mais inclusiva, viabilizando um atendimento de qualidade, além de formar profissionais mais humanizados.

\section{REFERÉNCIAS}

ARAÚJO, A.M et al. A dificuldade no atendimento médico às pessoas surdas. Revista Interdisciplinar Ciências Médicas. 2019; 3(1): 3-9.

ARAÚJO, A.S.; MENEZES, A.M.C.; ARAÚJO, A.C.S. A Educação de Surdos: Formação de Professores na Língua Brasileira de Sinais (LIBRAS). Id on Line Rev Mult Psic. n. 11, v. 38, 2017.

BRASIL. Instituto Brasileiro de Geografia e Estatística. Censo Brasileiro de 2010. Rio de Janeiro: IBGE, 2012.

BRASIL. Decreto n. 5.626, de 22 de dezembro de 2005. Regulamenta a Lei n. 10.436 de 24 de abril de 2002, que dispõe sobre a Língua Brasileira de Sinais - Libras, e o art. 18 da Lei n. 10.098, de 19 de dezembro de 2000. Diário Oficial da República Federativa do Brasil, Brasília, 22 dez. 2005.

CAVAGNA, V.M et al. O paciente surdo e suas vivências no sistema de saúde: uma interface com a enfermagem. Revista Enfermagem Atual InDerme. n. 80, v.18, p. 33-39, 2019. https://revistaenfermagematual.com/index.php/revista/article/view/345 
CFN - Conselho Federal de Nutricionistas. Resolução CFN no 599, de 25 de fevereiro de 2018. Aprova o código de ética e conduta do nutricionista e dá outras providências. Diário Oficial da União. Brasília, n. 64, p. 182, 2018.

CUNHA, R.P.S.; PEREIRA, M.C.; OLIVEIRA, M.L.C. Enfermagem e os cuidados com pacientes surdos no âmbito hospitalar. REVISA. n. 8, v. 3, p. 367-77, 2019.

FRANÇA, E.G et al. Dificuldade de profissionais na atenção à saúde da pessoa com surdez. Ciências y Enfermaria XXII. n. 22, v. 3, p. 107-116, 2016. https://scielo.conicyt.cl/pdf/cienf/v22n3/0717-9553-cienf-22-03-00107.pdf

GOMES, L.F et al. Conhecimento de Libras pelos Médicos do Distrito Federal e Atendimento ao Paciente Surdo. Revista Brasileira de Educação Médica. n. 41, v. 4, p. 551-556, 2017.

GUEDES, V.N.; SILVA, A.F.A. Avaliação das condições de acessibilidade em edificações públicas de serviço de assistência social em Recife-PE. Revista Nacional de Gerenciamento de Cidades. n. 05, v. 29, 2017.

JALLES, M.P.; SANTOS, V.S.J.; REINALDO, A.M.S. Análise da produção científica sobre comunicação terapêutica no campo da saúde, saúde mental e álcool e outras drogas. Rev Med. n. 96, v. 4, p. 232-240, 2017.

KASTRUP, V.; THOMA, A.S. Inclusão e Acessibilidade para Pessoas com Deficiência. Psicologia: Ciência e Profissão. n. 38, v. 3, p. 551-553, 2018. https://doi.org/10.1590/1982-3703000102018

MACHADO, W.C.A et al. Comunicação de profissionais de unidade de reabilitação física com clientes surdos. Revista da Rede de Enfermagem do Nordeste. n. 16, v. 5, p. 649655, 2015. http://periodicos.ufc.br/rene/article/view/2781

MARQUETE, V.F.; COSTA, M.A.R.; TESTON, E.F. Comunicação com deficientes auditivos na ótica de profissionais de saúde. Rev Baiana Enferm. n. 32:e24055, 2018.

MOURA, A.F.; LEITE, L.P.; MARTINS, S.E.S.O. Possibilidades de acesso à Universidade: estudantes surdos em questão. Journal of Research in Special Educational Needs. $\mathrm{n}$. 16, n. 1, p. 876-879, 2016.

SANTOS, A.P.M.; BERGOLD, L.B. Oficinas musicais: a utilização do lúdico e da música para Educação Alimentar e Nutricional com escolares. RASBRAN - Revista da Associação Brasileira de Nutrição. n. 9, v. 2, p. 87-92, 2018.

SILVA, N.G.P.S.; ANDRADE, E.G.S. Comunicação eficaz através da língua brasileira de sinais do profissional de enfermagem com os deficientes auditivos. Rev Inic Cient Ext. $n$. 1, n. 1, p.11-7, 2018.

SOUZA, M.F.N.S et al. Principais dificuldades e obstáculos enfrentados pela comunidade surda no acesso à saúde: uma revisão integrativa de literatura. Revista CEFAC. n. 19, v. 3, p. 395-405, 2017. 
SOUZA, M.T.; PORROZZI, R. Ensino de libras para os profissionais de saúde: uma necessidade premente. Revista Práxis. n. 1, v. 2, p. 43-46, 2017. http://revistas.unifoa.edu.br/index.php/praxis/article/view/1119/1007

PIRES, H.F.; ALMEIDA, M.A.P.T. A percepção do surdo sobre o atendimento nos serviços de saúde. Revista Enfermagem Contemporânea. n. 5, v. 1, p. 68-77, 2016. 